\title{
Analytic Perturbation Theory Model for QCD and Upsilon Decay
}

\author{
D. V. Shirkov \\ Joint Institute for Nuclear Research, Dubna, 141980, Russia
}

\begin{abstract}
An elegant and more precise (Denominator) formula for the 3-loop perturbative QCD coupling is discussed. It improves the common expression (e.g., canonized by $\mathrm{PDG}$ ) in few $\mathrm{GeV}$ region. On its base, we propose simple analytic Model for ghostfree QCD running couplings and their effective powers within the Analytic Perturbation Theory, in both the space-like (Euclidean) and time-like (Minkowskian) regions, very accurate in the range above $1 \mathrm{GeV}$.

Effectiveness of the new Model is illustrated by the example of $\Upsilon(1 \mathrm{~S})$ decay where the standard analysis gives $\alpha_{s}\left(M_{\Upsilon}\right)=0.170 \pm 0.004$ value that is inconsistent with the bulk of data for $\alpha_{s}$. Instead, we obtain $\alpha_{s}^{M o d}\left(M_{\Upsilon}\right)=0.185 \pm 0.005$ that corresponds to $\alpha_{s}^{M o d}\left(M_{Z}\right)=0.120 \pm 0.002$ that is close to the world average.
\end{abstract}

\section{Introduction}

This text contains essence of three topics related to the QCD coupling at low energy:

1. Presenting of QCD perturbative coupling $\alpha_{s}(\mu)$ by Denominator representation instead of "canonical" PDG-like bulky expression.

2. Particular "Analytic-Perturbation-Theory" (APT) ghost-free model for $\alpha_{s}(\mu)$ in the low-energy region.

3. APT Model analysis of Upsilonium decay.

It is based mainly upon recent paper [1] which contain more detailed exposition.

\section{$1.1 \quad$ Notation}

We use the Bethke[2] notation (different from the PDG one)

$$
\frac{d \alpha_{s}}{d L}=\beta\left(\alpha_{s}\right)=-\beta_{0} \alpha_{s}^{2}-\beta_{1} \alpha_{s}^{3}-\beta_{2} \alpha^{4}+\ldots, \quad L=\ln \left(x / \Lambda^{2}\right),
$$

for beta-function coefficients $\beta(\alpha)=-\beta_{0} \alpha^{2}\left(1+b_{1} \alpha+b_{2} \alpha^{2}+\ldots\right) ; b_{k}=\beta_{k} / \beta_{0}$,

$$
\beta_{0}\left(n_{f}\right)=\frac{33-2 f}{12 \pi} ; \quad b_{1}\left(n_{f}\right)=\frac{153-19 f}{2 \pi(33-2 f)} ; \quad b_{2}^{\overline{\mathrm{MS}}}\left(n_{f}\right)=\frac{2857-(5033 / 9) n_{f}+(325 / 27) n_{f}^{2}}{32 \pi^{2}\left(11-(2 / 3) n_{f}\right)}
$$


Here, numerically, all $\beta_{k}, b_{k}$ and $B=\beta_{1}^{2} / \beta_{0}$ are of an order of unity

$$
\begin{gathered}
\beta_{0}(4 \mp 1)=0.6631 \pm 0.0530 ; \quad \beta_{1}(4 \mp 1)=0.3251 \pm 0.0802 ; \quad \beta_{2}(4 \mp 1)=0.205_{-0.112}^{+0120} ; \\
b_{1}(4 \pm 1)=0.4902_{+0.0757}^{-0.0889} ; \quad b_{2}(4 \mp 1)=0.309_{+0.144}^{-0.159} ; \quad B(4 \mp 1)=0.7392_{-0.0814}^{+0.0509} .
\end{gathered}
$$

\section{Perturbative QCD coupling}

\subsection{Denominator representation for 3-loop $\alpha_{s}(\mu)$}

Instead of canonical ( Bethke[2], PDG[3]) 1/L expanded expression

$$
\begin{gathered}
\bar{\alpha}_{s}^{(3)}(x)=\frac{1}{\beta_{0} L}-\frac{b_{1}}{\beta_{0}^{2}} \frac{\ln L}{L^{2}}+\frac{1}{\beta_{0}^{3} L^{3}}\left[b_{1}^{2}\left(\ln ^{2} L-\ln L-1\right)+b_{2}\right]- \\
-\frac{1}{\beta_{0}^{4} L^{4}}\left[b_{1}^{3}\left(\ln ^{3} L-\frac{5}{2} \ln ^{2} L-2 \ln L+\frac{1}{2}\right)+3 b_{1} b_{2} \ln L-\frac{b_{3}}{2}\right]+\ldots \quad\left(9.5_{P D G}\right)
\end{gathered}
$$

we argue to use the "Denominator representation" for $\alpha_{s}(\mu)$

$$
\bar{\alpha}_{s}^{(3, i t e r)}(L)=\frac{1}{\beta_{0} L+b_{1} \ln \left(L+\frac{b_{1}}{\beta_{0}} \ln L\right)+\frac{\left(b_{1}^{2}-b_{2}\right)}{\beta_{0} L}+\frac{b_{3}}{2\left(\beta_{0} L\right)^{2}}} ; \quad L=\ln \frac{\mu^{2}}{\Lambda^{2}}
$$

Canonical form can be obtained from it by use of casual expansion parameter $\epsilon_{\mathbf{2}}$

$$
\begin{gathered}
\bar{\alpha}_{s}^{(3, i t e r)}(L)=\frac{1}{\beta_{0} L}\left(1+\frac{B \ln [L+B \ln L]}{L}+\varepsilon_{2}^{2}-\varepsilon_{3}+\frac{\varepsilon_{4}}{2}\right)^{-1} \simeq \\
\frac{1}{\beta_{0} L}\left[1+\left(\epsilon_{\mathbf{2}}+\epsilon_{\mathbf{2}} \varepsilon_{2}+\varepsilon_{2}^{2}\right)-\left(\varepsilon_{3}+\frac{\epsilon_{\mathbf{2}} \varepsilon_{2}}{2}\right)+\ldots\right]^{-1} ; \quad \epsilon_{\mathbf{2}}=\frac{B \ln L}{L} \ll 1
\end{gathered}
$$

as well as the higher loops ones: $\varepsilon_{k+1}=\frac{b_{k}}{\left(\beta_{0} L\right)^{k}}(k \geq 1)$. For instance, at $n_{f}=3,4$

$\epsilon_{2}(3 \div 4)=[0.79 \div 0.74] \frac{\ln L}{L} ; \quad \varepsilon_{2}(3 \div 4)=\frac{[0.79 \div 0.74]}{L} ; \quad \varepsilon_{3}(3 \div 4)=\left(\frac{0.90 \div 0.84}{L}\right)^{2} ; \quad \varepsilon_{4}=\left(\frac{1.2}{L}\right)^{3}$.

Then, $\epsilon_{\mathbf{2}}{ }^{2} \sim \varepsilon_{3}$ at $\ln L^{*} \sim 1.13: L^{*} \sim 3.3$, that is at $Q^{*} \sim 5.0 \Lambda, \quad Q \sim 1.6 \div 2 \mathrm{GeV}$.

There, $\epsilon_{\mathbf{2}} \sim 0.25, \varepsilon_{3} \sim 0.07$. Relative $\left(9.5_{P D G}\right)$ error $\sim \epsilon_{\mathbf{2}}{ }^{3} \sim 0.02$, while error of $(2)$ $\sim \varepsilon_{4} / 2 \simeq 0.03$ Ghost singularity at $L=0$ in (2) is much weaker than, in $\left(9.5_{P D G}\right)$. 


\subsection{The beta function arbitrariness}

In turn, Denominator representation (2) is an iterative approximate solution of RG differential eq.(1) quadrature which, in the two-loop (NLO) case, is

$$
\beta_{0} L=-\int^{\alpha_{s}} \frac{d a}{a^{2}\left(1+b_{1} a\right)}=\frac{1}{\alpha_{s}}+b_{1} \ln \frac{\alpha_{s} \beta_{0}}{1+b_{1} \alpha_{s}}
$$

- a transcendental relation that can be resolved[4] in terms of special Lambert function. Usually, instead, one solves this relation iteratively with the result

$$
\alpha_{s}^{2, \text { iter }}(L) \simeq \frac{1}{\beta_{0} L+b_{1} \ln \left(B+1 / \alpha_{s}^{(1)} \beta_{0}\right)}=\frac{1}{\beta_{0} L+B \beta_{0} \ln (L+B)}
$$

with its famous log-of-log dependence.

Meanwhile, if one transforms 2-loop beta function á la Padé $\beta_{2} \rightarrow \beta_{2}^{P}(\alpha)=-\frac{\beta_{0} \alpha^{2}}{1-b_{1} \alpha}$, the result of integration and iteration will be different

$$
\beta_{0} L=-\int^{\alpha_{s}} \frac{d a}{a^{2}}\left(1-b_{1} a\right)=\frac{1}{\alpha_{s}}+b_{1} \ln \left(\beta_{0} \alpha_{s}\right), \quad \alpha_{s}^{2 P, i t e r}(L)=\frac{1}{\beta_{0} L+b_{1} \ln L} .
$$

Thus, the inner self-consistency condition of the 2-loop (NLO) approximation looks like

$$
\frac{\triangle \alpha^{(2)}}{\alpha^{(2)}}=\frac{\alpha^{(2 P)}-\alpha^{(2)}}{\alpha^{(2)}} \simeq \varepsilon_{2}^{2} \ll 1
$$

Due to this, the intrinsic accuracy estimate for the two-loop QCD coupling $\alpha^{(2)}$ at $1.5-2 \mathrm{GeV}$ region is about $7 \%$, that is (in the $\overline{\mathrm{MS}}$ scheme) equal to the value of three-loop contribution $\sim \varepsilon_{3}$. At the same time, at the b-quark mass $\sim 4.5 \mathrm{GeV}$ one has $\varepsilon_{2}^{2} \simeq 0.015, \epsilon_{\mathbf{2}} \sim 0.21, \epsilon_{\mathbf{2}}{ }^{3} \sim 0.01$

This means that in the $n_{f}=4,5$ domain one can use only the first line of the 3-loop eq. $\left(9.5_{P D G}\right)$ and second expression (5) instead of (2) with the same accuracy.

Numerically, at 4-flavor region for $\Lambda_{4} \sim 300 \mathrm{MeV}$ and $q=1.5 ; 3 \mathrm{GeV}$ one has $\varepsilon_{2}(q=$ $1.5 \mathrm{GeV}) \sim 0.053, \quad \epsilon_{\mathbf{2}}{ }^{4}(1.5) \sim 0.020, \varepsilon_{2}(3) \sim 0.025, \quad \epsilon_{\mathbf{2}}{ }^{4}(3) \sim 0.020$ and at 5-flavors for $\Lambda_{5} \sim 225 \mathrm{MeV} ; \quad q \sim 4.5 \mathrm{GeV}-\varepsilon_{2}(4.5) \sim 0.012, \epsilon^{2}(4.5) \sim 0.030$.

Pragmatically, this means that, within the 2 per cent limit of accuracy, one can equally use simple two-term denominator eq.(5) up to $3 \mathrm{GeV}$ scale. However, in the $1 / L$-expanded form one needs to keep 3 terms (of expansion in powers of $\epsilon_{\mathbf{2}}$ ) in $n_{f}=5$ region and 5 terms in the $n_{f}=4$ one.

As it can be shown, the inner consistency of the 3-loop iterative approximations is controlled by the same parameters $\varepsilon_{2}$ and $\epsilon_{\mathbf{2}}$. At the same time, the measure for importance of direct 3-loop (NNLO) term $\varepsilon_{3}=b_{2} /\left(\beta_{0} L\right)^{2}$ should be compared with $\varepsilon_{2}$. It turns out that they are close (within 40 per cent) to each other. 


\section{Analytic Perturbation Theory}

\subsection{Outline of Analytic Perturbation Theory}

Remind first, that the cornerstones of APT are the $Q^{2}$ analyticity of coupling functions and compatibility with linear integral transformations. For the fresh reviews of APT see [5, 6].

Here follows compendium of main definitions. The most elegant APT formulation is based on the set of spectral functions $\left\{\rho_{i}(\sigma)\right\}$ defined as

$$
\rho_{k}(z)=\operatorname{Im}\left(\left[\alpha_{s}(-z)\right]^{k}\right) .
$$

The first of them, $\rho_{1}=\rho(\sigma)$ is just the Källen-Lehmann spectral density for the Euclidean APT coupling. Then, higher Euclidean ("analyticized $k$ th power of coupling in the Euclidean domain") and Minkowskian ("effective $k$ th power of coupling in the Minkowskian domain") APT functions will be

$$
\mathcal{A}_{k}\left(Q^{2}\right)=\mathbb{A}\left[\alpha_{s}^{k}\right]=\frac{1}{\pi} \int_{0}^{+\infty} \frac{\rho_{k}(\sigma) d \sigma}{\sigma+Q^{2}} ; \quad \mathfrak{A}_{k}(s)=\mathbb{R}\left[\alpha_{s}\right]=\frac{1}{\pi} \int_{s}^{+\infty} \frac{d \sigma}{\sigma} \rho_{k}(\sigma) .
$$

They are related by integral transformation and satisfy differential relations

$$
\mathcal{A}_{k}\left(Q^{2}\right)=\mathbb{D}\left[\mathfrak{A}_{k}\right]=Q^{2} \int_{0}^{+\infty} \frac{\mathfrak{A}_{k}(s) d s}{\left(s+Q^{2}\right)^{2}} ; \quad \frac{1}{k} \frac{d \mathfrak{A}_{k}(s)}{d \ln s}=-\sum_{n \geq 1} \beta_{n-1} \mathfrak{A}_{k+n}(s),
$$

which can be used for iterative definitions.

\subsection{Properties of the APT functions}

For the one-loop case, the APT formulae are simple and elegant. Starting with the perturbative RG-improved QCD coupling $\alpha_{s}^{(1)}\left(Q^{2}\right)=1 /\left(\beta_{0} l\right)$, with the help of (7), (8) one arrives at the ghost-free effective Euclidean and Minkowskian 1

$$
\mathcal{A}_{1}^{(1)}(l)=\frac{1}{\beta_{0}}\left(\frac{1}{l}-\frac{1}{e^{l}-1}\right) ; \quad \mathfrak{A}_{1}^{(1)}(L)=\frac{1}{\beta_{0} \pi} \arccos \left(\frac{L}{\sqrt{L^{2}+\pi^{2}}}\right)
$$

APT couplings. Higher functions $\mathcal{A}_{i}, \mathfrak{A}_{i}$ can be defined via recursive relations (9) with only one term in the r.h.s. For instance, Minkowskian functions are

$$
\mathfrak{A}_{2}^{(1)}(L)=\frac{1}{\beta_{0}^{2}} \frac{1}{L^{2}+\pi^{2}}, \quad \mathfrak{A}_{3}(L)=\frac{1}{\beta_{0}^{3}} \frac{L}{\left(L^{2}+\pi^{2}\right)^{2}}, \quad \mathfrak{A}_{4}(L)=\frac{1}{\beta_{0}^{4}} \frac{L^{2}-\pi^{2} / 3}{\left(L^{2}+\pi^{2}\right)^{3}} .
$$

\footnotetext{
${ }^{1}$ Here, we change arguments of the APT functions: $Q^{2} \rightarrow l=\ln \left(Q^{2} / \Lambda^{2}\right)$ and $s \rightarrow L=\ln \left(s / \Lambda^{2}\right)$.
} 
Higher-loop case. The two-loop expressions are more complicated. Here, exact QCD coupling $\alpha_{s}$ can be expressed explicitly in terms of a special Lambert function $W$ defined as a solution of the transcendental equation $W(z) e^{W}=z$. This expression yields rather complicated formulae for $\alpha_{E}=\mathcal{A}_{1}$ and $\alpha_{Q}=\mathfrak{A}_{1}$ in terms of the main branch $W_{-1}$.

At the three-loop case, one meets further complications. Here, only for Padé approximated beta-function, exact solution can be expressed [4] in terms of the Lambert function. Such expression are not comfortable enough for practical use.

The devised scheme with due account for matching[7], known as "global APT", has been studied numerically by Magradze and Kourashev at the two- and three-loop level. They calculated numerical tables for the first three functions $\mathfrak{A}_{k} 1,2,3$ and $\mathcal{A}_{1,2,3}$ at three values of $\Lambda^{n_{f}=3}=350,400,450 \mathrm{MeV}$ in the interval $1 \mathrm{GeV}<\sqrt{s}, Q<100 \mathrm{GeV}$ [4, 8], and $\mathfrak{A}_{1,2}, \mathcal{A}_{1,2}$ in the interval $0.1 \mathrm{GeV}<\sqrt{s}, Q \lesssim 3 \mathrm{GeV}[9]$.

The APT functions obey important properties valid in the higher-loop case:

- In the Euclidean and Minkowskian domains, QCD couplings $\alpha_{E}\left(Q^{2}\right)=\mathcal{A}_{1},\left(Q^{2}\right)$, $\alpha_{M}(s)=\mathfrak{A}_{1}$ and their "effective powers" $\mathcal{A}_{k}\left(Q^{2}\right) \mathfrak{A}_{k}(s)$, are different functions related by integral operations $\mathbb{A}[]$ and $\mathbb{R}[]$ explicitly defined in eqs. (8). Higher functions like (8),(9), are not equal to powers of the first ones (10).

- The APT functions $\mathcal{A}_{k}\left(Q^{2}\right) \mathfrak{A}_{k}(s)$ differ of common expansion functions $\left(\alpha_{s}\right)^{k}$ in the low energy region, where they are regular with finite $\alpha_{E}(0)=\alpha_{M}(0)=1 / \beta_{0}$ or zero limits. Unphysical singularities are absent with no additional parameters introduced. This behavior provides high stability with respect to change of renormalisation scheme [10. In the UV limit, all APT functions tends to their usual counterparts $\left(\alpha_{s}\right)^{k}$.

- Expansion of an observable in coupling powers $\left(\alpha_{s}\left(Q^{2}\right)\right)^{n}$ for the Euclidean or in $\left(\alpha_{s}(s)\right)^{n}$ for the Minkowskian case is substituted by nonpower expansion in sets $\left\{\mathcal{A}_{k}\right\}$, or $\left\{\mathfrak{A}_{k}\right\}$ respectively. The latter expansions exhibit a faster convergence.

The APT re-examination of various processes has been performed in number of papers [11 - [14]. In particular, paper [11] contained the first attempt in revising the $\Upsilon$ decay. Simple estimates for the influence of $\pi^{2}$-terms upon some observables were performed in [5]. The $\tau$ decay was re-examined in [12]. Pion form factor was studied [13, 14] within the APT techniques. One more extraction of APT coupling at $Q \sim 100-400 \mathrm{MeV}$ was made 15 by the Milano group from mass spectrum analysis of ground and first excited quarkonium states. 


\section{Simple Model for 3-Loop APT Functions}

\section{1 "One-Loop-Like" Model}

Our aim is to construct simple and accurate enough (for practical use) analytic approximations for two sets of functions $\mathfrak{A}_{k}$ and $\mathcal{A}_{k}, k=1,2,3$. To reduce number of fitting parameters, one should better provide the applicability of the recurrent relations. To this goal, we use one-loop APT expressions, eqs.(10) - (11), with modified logarithmic arguments

$$
\mathcal{A}_{k}^{\text {mod }}(l)=\mathcal{A}_{k}^{(1)}\left(l_{*}\right) ; \quad \mathfrak{A}_{k}^{\bmod }(L)=\mathfrak{A}_{k}^{(1)}\left(L_{*}\right),
$$

$L_{*}$ and $l_{*}$ being some "two-loop RG times". Model functions (12) are related by the "one-loop-type" recursive relations

$$
\mathcal{A}_{n+1}^{\text {mod }}=-\frac{1}{n \beta_{0}} \frac{d \mathcal{A}_{n}^{\text {mod }}}{d l_{*}}=-\frac{1}{n \beta_{0}} \frac{d \mathcal{A}_{n}^{\text {mod }}}{d l} \cdot \frac{d l}{d l_{*}}, \quad \mathfrak{A}_{n+1}^{\text {mod }}=-\frac{1}{n \beta_{0}} \frac{d \mathfrak{A}_{n}^{\text {mod }}}{d L_{*}} .
$$

A simple expression for $l_{*}$ was taken from [16], where a plain approximation for the two-loop effective $\log l_{2}=l+b \ln \sqrt{l^{2}+4 \pi^{2}}$, with $b$ defined in Section 1 was used. This approximation combined reasonable accuracy in the low-energy range with the absence of singularities for $\alpha_{E}$. We extend this approach to higher functions in both the Euclidean and Minkowskian domains and change square root in "effective logs" $L_{2}(a)$ and $l_{2}(a)$ : $\sqrt{l^{2}+4 \pi^{2}} \rightarrow \sqrt{l^{2}+a \pi^{2}}$ with $a$, an adjustable parameter. It comes out from thorough numerical analysis that optimal value of the new parameter is $a \approx 2$, while effective boundaries between the flavor regions have to be chosen on quark masses $m_{c}=1.3 \mathrm{GeV}$ and $m_{b}=4.3 \mathrm{GeV}$ just as in the $\overline{M S}$ scheme.

That is, our Model consists of a set of equations (13) with (10) - (12) and

$$
L_{*}=L_{2}(a=2)=L+B \ln \sqrt{L^{2}+2 \pi^{2}}, \quad l_{*}=l_{2}(2)=l+B \ln \sqrt{l^{2}+2 \pi^{2}} .
$$

Here, $L$ and $l$ contain common $\Lambda_{\overline{\mathrm{MS}}}$ values, like in (10), for each of the flavor region. Advantage of Model (10)-(11), (12), (13) is that it involves only one new parameter, $a=2$ with $\Lambda_{\overline{\mathrm{MS}}}$ and $n_{f}$ taking their usual values.

\subsection{Accuracy of the Model vs data errors}

In paper [1] errors of Model expressions (11), (12), (13) in each $n_{f}$ range were estimated

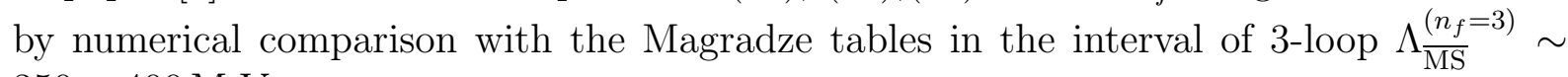
$350-400 \mathrm{MeV}$.

As it follows from that analysis, errors of Model for the first three APT functions are small, being of an order of 1-2 per cent for the first functions, of 3-5\% for the second and of $6-10 \%$ for the third ones in the region above $1.5 \mathrm{GeV}$, i.e., in the $n_{f}=4,5$ ranges. However, its accuracy in the $n_{f}=3$ region (above $1 \mathrm{GeV}$ ) is at the level of 5-10 per cent. 
Meanwhile, relative contributions of typical LO, NLO and NNLO terms in APT nonpower expansion for observables are usually something like $60-80 \%, 30-10 \%$, and $10-1 \%$, respectively (see Table 2 in Ref.[5]). Due to this, the Model accuracy for many cases is defined by that of the first model functions $\mathcal{A}_{1}^{\text {mod }}, \mathfrak{A}_{1}^{\text {mod }}$, provided that QCD contribution to an observable starts from one-loop contribution $\sim \alpha_{s}$. At the same time, for quarkonium decays the leading contribution $\sim \alpha_{s}^{3}$. There, the Model error is defined by accuracy of the third Minkowskian function $\mathfrak{A}_{3}^{\bmod }$.

In the Table 2 of paper [1] we compared Model errors with some data errors in the low energy region.

With due regard for data errors, we can now set some total margin of accuracy that our Model would satisfy. This margin may be chosen, e.g., as $1 / 3$ of the data error bar, which is no less than $10 \%$. Then, the accuracy limit, imposed on the first APT functions could be about $3 \%$, for the second function ca $10 \%$, and for the third ones, about $20 \%$. Due to this, it is not reasonable to use the Model below $0.5 \mathrm{GeV}$, whereas above this limit it is fully advisable. Now, we proceed to its practical application to $\Upsilon$ decay.

\section{$5 \Upsilon(1 \mathrm{~S})$ Decay Revised}

\section{$5.1 \Upsilon$ Widths}

Our first motive for this choice is that the parameter $\epsilon_{M}(L)=\pi^{2} / L^{2}$ responsible for deviation of APT Minkowskian functions from powers of canonical $\alpha_{s}$, is not very small in the region $(5-10 \mathrm{GeV})$ related to this decay: $\epsilon_{M}(L) \simeq 0.16-0.27, \quad \epsilon_{M}\left(L_{2}\right) \simeq 0.11-0.18$.

Another processes involving $\Upsilon$, are $\Upsilon$ radiative decays and $\Upsilon$ production. But they have low data precision (about 10\% - 40\%). Besides, the non-radiative decay of $1 S$-state provides the best data precision (1.5\% for the ratio of hadronic and leptonic widths [3]).

The second arguument is the disagreement with the world average.

For an extensive review on $q \bar{q}$ decay widths see [17]. NLO ratio of hadronic and leptonic decay widths of $S$ state of the $\Upsilon$ was given in [18]. However, their expression is not renorm-invariant. In our analysis, to return it to the RG-invariant form, we put scale parameter $\mu=M_{\Upsilon}$. Then

$$
R\left(s_{\Upsilon}\right)=\frac{\Gamma(\Upsilon \rightarrow \text { hadrons })}{\Gamma\left(\Upsilon \rightarrow e^{+} e^{-}\right)}=\frac{10\left(\pi^{2}-9\right) \alpha_{s}^{3}\left(s_{\Upsilon}\right)}{9 \pi \alpha^{2}\left(M_{\Upsilon}\right)}\left(1+\frac{\alpha_{s}\left(s_{\Upsilon}\right)}{\pi} 7.2\right), \quad s_{\Upsilon}=M_{\Upsilon}^{2} .
$$

Then, the issue of scale should be readdressed to choice of $s_{\Upsilon}$.

\subsection{Reevaluation of $\Lambda_{Q C D}$ from $\Upsilon$ Decay}

First attempt to evaluate $\Lambda_{Q C D}$ extracted from $\Upsilon$ decay by proper taking into account analytic continuation effects was made in [11. Analogous analysis is performed here, 
employing better accuracy Model expression for $\mathfrak{A}_{i}(s)$ and using more fresh data from CLEO III detector [19] as well as APT expansion instead of formula (14), that, within our Model, is reduced to

$$
R_{\Upsilon}(s)=5360\left[\mathfrak{A}_{3}(L)+\frac{\mathfrak{A}_{4}(L)}{\pi} 7.2\right] \quad \rightarrow \quad 5360\left(\mathfrak{A}_{3}^{(1)}\left(L_{2}\right)+2.30 \mathfrak{A}_{4}^{(1)}\left(L_{2}\right)\right) .
$$

We extracted by this formula $\Lambda \frac{(5)}{\mathrm{MS}}$ and $\alpha_{s}$ values, from fresh data $R_{\Upsilon}=37.3 \pm 0.75[19]$. In Table, results, obtained within APT are compared to results of standard PT. In line

Table 1: Results of various $\alpha_{s}$ extraction from Upsilon decays

\begin{tabular}{|l|c|c|c|}
\hline \multicolumn{4}{|c|}{ Part I. Non-APT treatment } \\
\hline \multicolumn{1}{|c|}{ Source } & $\alpha\left(M_{\Upsilon}\right)$ & $\alpha\left(M_{Z}\right)$ & $\Lambda_{\overline{\mathrm{MS}}}^{n_{f}=5}$ \\
\hline PDG, $\Upsilon, 1 S$ & $0.170(4)$ & $0.112(2)$ & $146_{-17}^{+18}$ \\
\hline PDG, global Fit & $0.182(5)$ & $0.1185(20)$ & $217_{-23}^{+25}$ \\
\hline \hline \multicolumn{4}{|c|}{ Part II. APT treatment } \\
\hline Exact APT, 1S & $0.1805(12)_{\exp }$ & $0.1179(5)_{\exp }$ & $210(5)$ \\
\hline [Mod], 1S & $\mathbf{0 . 1 8 5}(5)_{\mathrm{M}}$ & $0.120(2)_{\mathrm{M}}$ & $235(25)$ \\
\hline [5] Crude APT & 0.183 & 0.119 & 222 \\
\hline
\end{tabular}

1 of Part I, "PDG, $1 S$ ", standard PT results on $\Upsilon(1 S)$ decay are given. We present them not exactly as they were published in [3] but recalculated along with modern experimental data. Line 2, marked "PDG, Fit" gives the published world average value described by the curve on Fig. 9.2 in [3], within the error bars of all the processes. Column " $\alpha\left(M_{\Upsilon}\right)$ " means " $\alpha_{s}$, calculated at the mass of $\Upsilon$, according to eq.(9.5) of [3]".

Line 1 of Part II, "Exact APT", presents results of $\Upsilon 1 \mathrm{~S}$ decay calculated by exact numeric tables for $\mathfrak{A}_{3}$ and by [20] for $\mathfrak{A}_{4}$ APT function. Line 2, [Mod], presents values obtained from $\Upsilon(1 S)$ decay data by means of the Model eqs.(12),(13). Here, model errors combine Model errors of both the terms in the r.h.s. of eq.(15). Line 3, "Crude APT" gives an earlier result [5] with crude APT estimate used to correct the Bethke-2000 value $\alpha_{s}\left(M_{\Upsilon}\right)=0.170$ extracted there from all the $\Upsilon$ decays data.

\section{Acknowledgements}

An essential part of this material is based on common work with Andrej Zayakin. The author is grateful also to A.Bakulev, A.Kataev, S.Mikhailov, A.Pivovarov for important advices, as well as to Roman Pasechnik for help in numerical calculation. The work has been supported in part by RFBR grant No. 05-01-00992 and by Scient.School grant 2339.2003.2. 


\section{References}

[1] D. V. Shirkov and A.V. Zayakin; hep-ph/0512325.

[2] S. Bethke, hep-ex/0004021, J. Phys. G, 26 R27 (2000); S. Bethke, hep-ex/0407021, Nucl.Phys.Proc.Suppl. 135, 345 (2004).

[3] S. Eidelman et al., Phys. Lett. B 592, 1 (2004).

[4] B. A. Magradze, "QCD coupling up to third order in standard and analytic perturbation theories", RMI-000-15 (2000); JINR-E2-2000-222 (2000); hep-ph/0010070.

[5] D. V. Shirkov, hep-ph/0107282; Eur. Phys. J. C 22, 331 (2001).

[6] D. V. Shirkov, in QCD@Work 2005 (Proceed. Int'nl Workshop on QCD, Conversano, Bari, July '05, edited by P.Colangelo et al., AIP Conf. Proceed. 806, pp. 97-103; hep-ph/0506050;

[7] D. V. Shirkov, hep-ph/0012283 Theor. Math. Phys. 127, 409 (2001).

[8] D. S. Kurashev and B. A. Magradze, hep-ph/0104142; Theor. Math. Phys. 135, 531 (2003)

[9] B. A. Magradze, "Practical techniques of analytic perturbation theory of QCD", Report RMI-2003-05; hep-ph/0305205.

[10] D. V. Shirkov and I. L. Solovtsov, hep-ph/9711251; Phys. Lett. B 442, 344 (1998).

[11] N. V. Krasnikov, A. A. Pivovarov, Phys. Lett. B 116, 168 (1982).

[12] O. P. Solovtsova, Theor. Math. Phys. 134, 365 (2003).

[13] A. P. Bakulev et al., hep-ph/0405062; Phys. Rev. D 70, 033014 (2004).

[14] A. P. Bakulev, S. V. Mikhailov, and N. G. Stefanis, hep-ph/0506311; Phys. Rev. D 72, 074014 (2005); A. P. Bakulev, A. I. Karanikas, and N. G. Stefanis, hep-ph/0504275 Phys. Rev. D 72, 074015 (2005).

[15] M. Baldicci and G. M. Prosperi, Phys. Rev. D 66, 074088 (2002); hep-ph/0412359, in AIP Conf. Proc. 756, pp. 152-161, (2005).

[16] I. L. Solovtsov and D. V. Shirkov, hep-ph/9909305; Teor. Mat. Fiz. 120 (1999) 482; [Theor. Math. Phys. 120, 1220 (1999)].

[17] G. T. Bodwin, E. Braaten, and G. P. Lepage, hep-ph/9407339 Phys. Rev. D 51, 1125 (1995)

[18] P. B. Mackenzie and G. P. Lepage, Phys.Rev.Lett., 47, 1244 (1981).

[19] G. S. Adams et al. [CLEO Collaboration],hep-ex/0409027; Phys. Rev. Lett. 94, 012001 (2005).

[20] B. Magradze, private communication. 